\title{
NOTES
}

\section{Preparation of Polymer Langmuir-Blodgett Multilayers Containing Axially Chiral Binaphthyl Group}

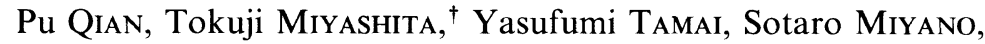 \\ and Minoru MATSUDA* \\ Department of Biochemistry and Engineering, Tohoku University, \\ Aoba Aramaki, Aoba-ku, Sendai 980, Japan \\ *Institute for Chemical Reaction Science, Tohoku University, \\ Katahira 2-1-1, Aoba-ku, Sendai 980, Japan \\ (Received May 13, 1991)
}

KEY WORDS Langmuir-Blodgett Multilayer / Monolayer / Axially Chiral / Binaphthyl / Poly $(N$-dodecylacrylamide) / Polymer LB Films /

The Langmuir-Blodgett (LB) technique was developed from the expectation of being applicable to microelectronics, molecular device, etc. ${ }^{1}$ Moreover, because the molecular layered structure in LB multilayer resembles the bilayer structure in biolipid membranes, biomimetic functionality such as molecular recognition, enantioselective permeation, and energy conversion, has been expected. ${ }^{2} \mathrm{We}$ have been interested in the preparation of LB multilayers of functional polymers which avoid poor thermal and mechanical stabilities of conventional LB multilayers of long-chain fatty acids. Recently we found that $N$ alkylacrylamide series have an excellent property to form monolayer and LB multilayer in both the monomer and polymer forms. ${ }^{3}$ In this work, optically active polymer LB multilayers (Y-type) were fabricated from monolayers of copolymers of $N$-dodecylacrylamide (DDA) with (S)-2-methoxy-1,1'-binaphthalene-2'-yl methacrylate ( $S$-BNMA). Described are the spreading behavior of the optical active polymer (DABM) on a water surface and preparation of the polymer LB multilayers.

\footnotetext{
${ }^{\dagger}$ To whom all correspondence should be addressed.
}

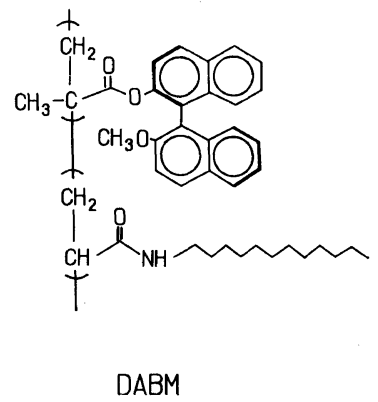

\section{RESULTS AND DISCUSSION}

The copolymers (DABM-1 and -2) of DDA with $S$-BNMA were prepared by conventional radical polymerization in benzene at $60^{\circ} \mathrm{C}$ with $2,2^{\prime}$-azobisisobutyronitrile as thermal initiator. The synthesis of $S$-BNMA is described elsewhere. ${ }^{4}$ The mole fractions of the $S$-BNMA unit were $0.08\left(\bar{M}_{n}=1.5 \times 10^{4}, \bar{M}_{w} / \bar{M}_{n}=1.84\right)$ and $0.11\left(\bar{M}_{n}=3.5 \times 10^{4}, \bar{M}_{w} / \bar{M}_{n}=1.52\right)$ for DABM-1 and -2 , respectively. The surface pressure - area isotherms of DABM-1 and -2 monolayers which were spread from a chloroform solution onto a water surface are shown in Figure 1 together with that of DDA 


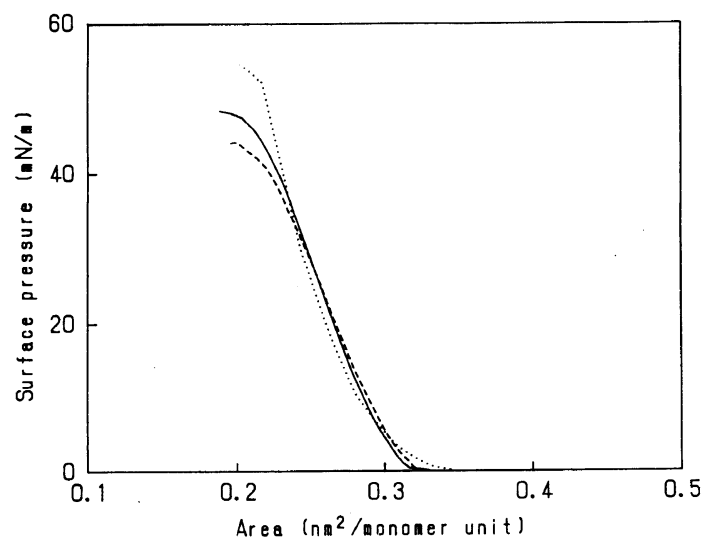

Figure 1. Surface pressure-area isotherms of copolymers (DABM-1 (-) and -2 (---)) and DDA homopolymer $(\cdots)$ at $15^{\circ} \mathrm{C}$.

homopolymer (PDDA). By the introduction of the $S$-BNMA unit into PDDA, the collapse pressure decreased. A steep rise in surface pressure and a high collapse pressure indicate, however, the formation of a stable condensed monolayer with. The average limiting surface area per monomer unit was estimated from the isotherms by extrapolating the steep rise in surface pressure to zero pressure. The surface area of DDA monomer unit in the homopolymer was found to be $0.28 \mathrm{~nm}^{2}$. From the mole fraction of $S$-BNMA and by assuming the area of DDA in the copolymers to be $0.28 \mathrm{~nm}^{2}$, the limiting surface area of $S$-BNMA monomer unit in DABM-1 and -2 was 0.56 and $0.54 \mathrm{~nm}^{2} /$ monomer unit, respectively. These values are in reasonable agreement with the cross section of the binaphthly group, as calculated from the CPK molecular model ( $c a$. $0.55 \mathrm{~nm}^{2}$, Figure 2). This means that the $S$-BNMA monomer unit fully occupy the surface.

The solid-like copolymer monolayers could be transferred successively onto solid supports such as quartz slides in both downward and upward strokes with a transfer ratio of 1.08 , giving the Y-type (bilayer structure) LB multilayer. The UV absorption and circular dichroism (CD) spectra of the multilayer

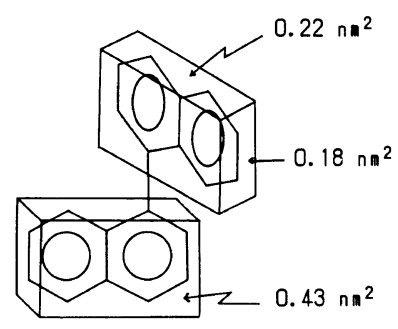

Figure 2. Cross section calculated from CPK model for binaphthyl group.

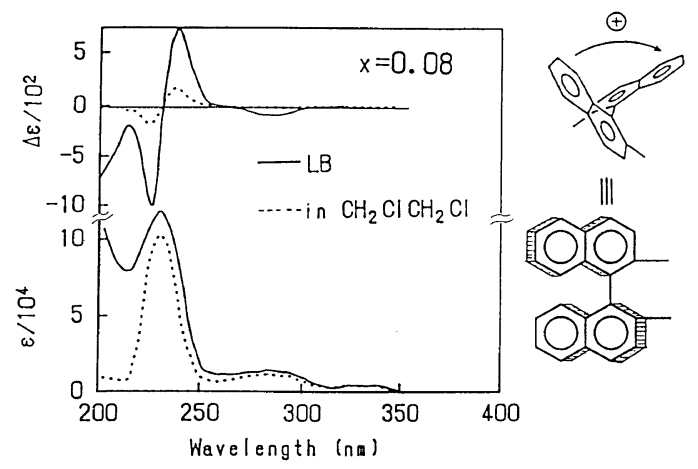

Figure 3. UV absorption (lower curve) and CD spectra (upper curves) of DABM-1 in LB multilayer (120 layers, $-)$ and 1,2-dichoroethane solution (---).

(DABM-1, 120 layers) are shown in Figure 3 together with those in 1,2-dichloroethane solution. The strong absorption at $228 \mathrm{~nm}$ is due to ${ }^{1} B_{\mathrm{b}}$ transition of naphthyl ring. The strong CD spectrum composed of positive first and negative second Cotton effects due to coupling of the ${ }^{1} B_{\mathrm{b}}$ transitions indicates $S$-configuration of the chiral binaphthyl moiety with a dihedral angle of around $90^{\circ} \mathrm{C} .{ }^{5}$ There is no shift in the CD spectrum for the $\mathrm{LB}$ multilayer, compared with that for 1,2dichloroethane solution, revealing that the chiral binaphthyl group is dispersed uniformly in the LB multilayer like in homogeneous solution. Apparently, a chiral chromophore is distributed uniformly in the LB assembly. Moreover, $\Delta \varepsilon$ in CD spectrum for the LB film is about five times as much as that at the same absorbance in 1,2-dichloroethane. This means that the binaphthyl chromophore is highly 
oriented in LB film.

Conclusively, although $S$-BNMA is nonamphiphilic, it is stably incorporated into multilayer. This is due to the excellent ability of the DDA unit to form monolayer. This method can be further developed for designing various LB multilayers. The axially chiral binaphthyl group apparently is capable of functioning as a chiral recognition site. We reported already that the $\mathrm{LB}$ multilayer of PDDA itself offers a suitable microenvironment for permselective membrane. ${ }^{6}$ Therefore, this chiral LB multilayer assembly is expected as a new type of functional ultrathin membrane with a chiral recognition ability. Its application to a permselective membrane for resolving optical isomers is currently under way.

\section{REFERENCES}

1. H. Kuhn, D. Möbius, and H. Bücher, "Physical Methods of Chemistry," Vol. 1, A. Weissberger and B. W. Rossiter, Ed., Wiley, New York, 1972, Part 3B, p 577.

2. J. H. Fendler, "Membrane Mimetic Chemistry," Wiley, New York, 1982.

3. T. Miyashita, H. Yoshida, T. Murakata, and M. Matsuda, Polymer, 28, 311 (1987); T. Miyashita, K. Sakaguchi, and M. Matsuda, Polym. Commun., 31, 461 (1990); T. Miyashita and M. Matsuda, Thin Solid Films, 168, L47 (1989); T. Miyashita, Y. Mizuta, and M. Matuda, Br. Polym. J., 22, 327 (1990); T. Miyashita, T. Yatsue, and M. Matsuda, J. Phys. Chem., 95, 2448 (1991).

4. Y. Tamai, P. Qian, K. Matsunaga, and S. Miyano, submitted.

5. N. Harada and K. Nakanishi, in "Circular Dichroic Spectroscopy-Exciton Coupling in Organic Stereochemistry," University Science Books, 1983.

6. T. Miyashita, M. Konno, M. Matsuda, and S. Saito, Macromolecules, 23, 3531 (1990). 